\title{
The nature of apoptosis of human breast cancer cells induced by three species of genus Ganoderma P. Karst. (Aphyllophoromycetideae) crude extracts
}

\begin{abstract}
The cytotoxic effect of crude extracts of three Ganoderma species (G. lucidum, G. tropicum, and G. tsugae) was screened against the human estrogen-independent cell line, MDA-MB435 using a microculture tetrazolium (MTT) assay. The crude extract obtained from the fruiting body of Ganoderma lucidum by hot water extraction procedures had IC50 values ranging from 140 to $350 \mathrm{gg} \mathrm{mLī} 1$. Acridine-orange/propidium iodine staining, electron microscopy, and transmission electron microscopy showed that the crude extract caused both apoptosis and necrosis. The G. lucidum mycelial crude extract was most effective, with an IC50 value of $148 \mathrm{\varepsilon g} \mathrm{mLī} 1$.
\end{abstract}

Keyword: Apoptosis; Ganoderma; IC50; Ling Zhi mushrooms; MDA-MB-435; Medicinal mushrooms; Mycelium; Necrosis 